\title{
Auscultation du glissement du versant ouest de la montagne Les Piniès dans la Drôme (France)
}

F. GIRAULT

BRGM

BP 6009

45060 Orléans Cedex 2
'Q D Différentes méthodes d'auscultation, certaines

expérimentales, ont été mises en ceuvre pour suivre

I'évolution du glissement du versant ouest de la

montagne des Piniès, dans la Drôme (France). Une carte

du champ des déformations superficielles du site et une description de l'évolution du glissement ont été obtenues par la comparaison d'ortho-images et modèles numériques de terrain (MNT) a très haute définition et par l'exploitation de systèmes de localisation par satellite. Les mesures, qui permettent de mieux décrire les phénomènes qui se déroulent dans ce versant, sont validées par un suivi topométrique classique.

\section{Remote sensing and monitoring of the ((Les Piniès )) landslide (Drôme, France)}

Monitoring methods, some of them being experimental, have been used to assess the $\alpha$ Les Pinies $x$ landslide (Dróme, France) A description of its evolution has been provided by satellite localisation systems; and by the comparison of high resolution Digital Elevation Models (DEMs) and ortho-images. A map of the superficial deformation field has been computed. Measurements are validated by a traditionnal topometric survey. 


\section{1}

\section{Introduction}

\section{1}

\section{Cadre}

Le mouvement de terrain dont il est question ici a été présenté lors des journées thématiques de ITNSA à Toulouse (A.M. Malatrait, 1995), et dans le numéro 75 de la Revue Française de Géotechnique (A.M. Malatrait, 1996). Il s'agit du glissement du versant ouest de la montagne des Piniès (Fig. 1), dans la commune de Boulc-enDiois (Drôme), dont les principales caractéristiques ne seront rappelées ici que brièvement. Le lecteur qui le souhaite pourra se reporter à l'article précédant dont la toponymie a été conservée. Rappelons seulement que, depuis 1977, un panneau calcaire de plus de 5 millions de $\mathrm{m}^{3}$ se détache en se disloquant de la montagne des Piniès (zone supérieure) et que d'importantes coulées de boue se produisent dans le fossé de Terre-Rouge Mondorès (zone médiane). Ces coulées constituent, dans la partie amont du vallon de Bonneval, un important cône d'épandage (zone inférieure).
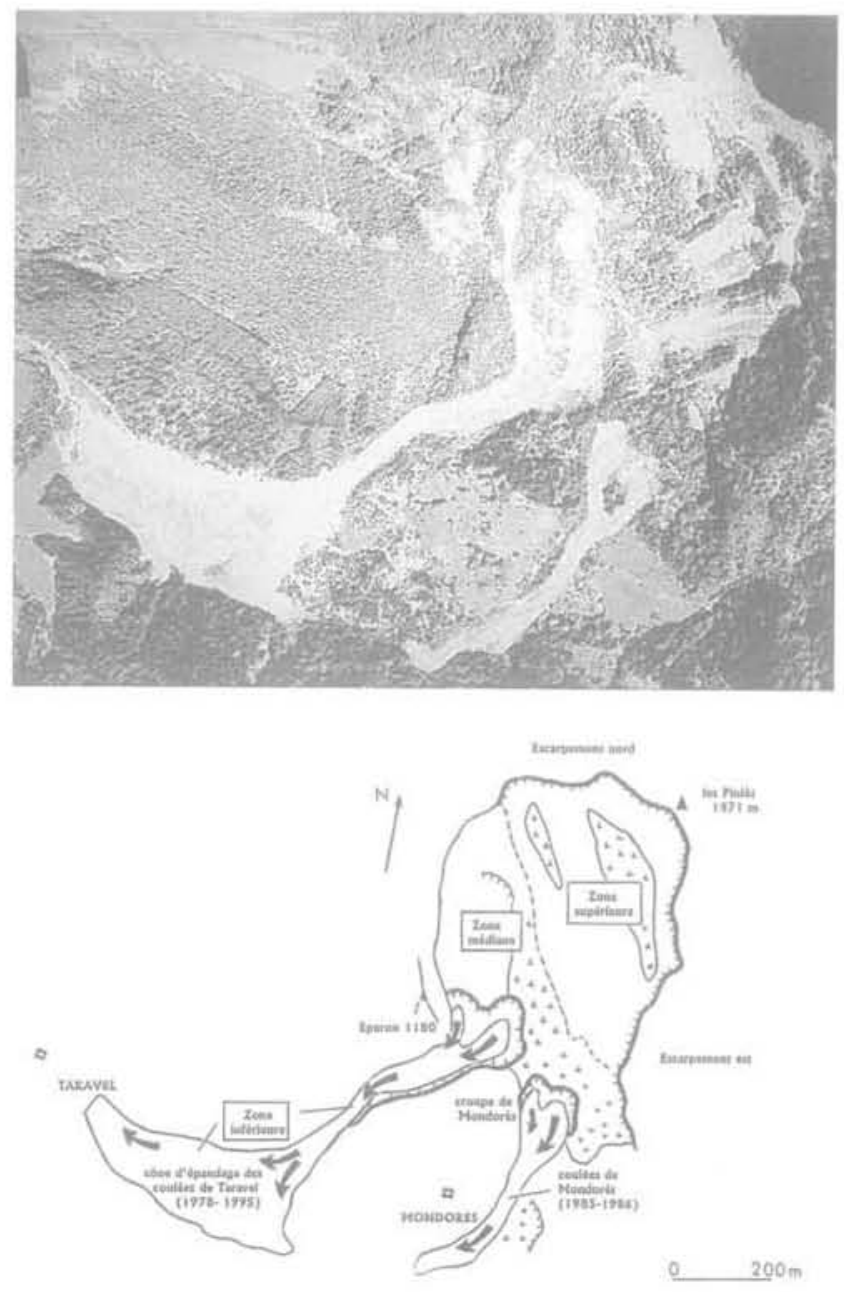

FIG.1 Le glissement du versant ouest de la montagne des Piniès; haut: ortho-image de 1993, bas : carte interprétative (d'après A.-M. Malatrait, 1992, modifié).

$\alpha$ Les Piniès $n$ landslide : up: 1993 ortho-image, down : interpretative map (after A.M. Malatrait (1992), modified).

\section{Objectifs}

L'ampleur et la diversité des phénomènes, très actifs, qui se déroulent dans ce versant en font un site idéal pour l'expérimentation de nouvelles méthodes d'auscultation. Dans le cadre de ses travaux de recherches, le BRGM, pour tenter de décrire l'évolution de ce site et d'en appréhender la dynamique, a effectué un suiví topométrique de ce glissement et mis en œuvre deux systèmes de positionnement par satellite: GPS (Global Positioning System) et DORIS (Doppler Orbitography and Radiopositioning Integrated by Satellite). Un examen comparatif d'images ortho-rectifiées et de modèles numériques de terrain (MNT) à mailles fines a également été réalisé, selon une méthode expérimentée avec succès sur 2 autres sites (Girault et Fleury, 1994, Girault, Goguel et Asté, 1995). Ces trois approches, à travers des données et des informations complémentaires, offrent une image plus complète de l'évolution du glissement.

\section{2}

\section{Méthodes d'auscultation}

\section{1}

\section{Suivi topométrique par visées optiques}

(27 janvier 1992-28 juin 1993)

\subsection{1. \\ Principe}

Un suivi topométrique par visées optiques (mesures des distances séparant un point de référence fixe de micro-réflecteurs) a été réalisé par l'IGN entre janvier 1992 et juin 1993 dans les zones médiane et supérieure.

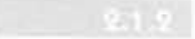 \\ Résultats}

Des vitesses moyennes et la direction de glissement du compartiment détaché de la montagne des Piniès ont été déterminées. Il apparait que l'ensemble des cibles se déplace de façon homogène vers le SW (direction moyenne N 225). Une forte accélération du glissement a été décelée entre mai 1992 et juin 1993:

- du 27 janvier au 20 mai $1992 \mathrm{v}=4,5 \mathrm{~mm} / \mathrm{jour}(1,6 \mathrm{~m} / \mathrm{an})$; - du 20 mai au 12 aoùt $1992 v=6 \mathrm{~mm} / \mathrm{jour}(2,2 \mathrm{~m} / \mathrm{an})$; - du 12 août au 28 juin $1993 \mathrm{v}=9,5 \mathrm{~mm} / \mathrm{jour}(3,5 \mathrm{~m} / \mathrm{an})$.

Ces déplacements se sont accompagnés de la dislocation progressive des masses calcaires affaissées.

\section{2}

Auscultation GPS (11 juin-15 novembre 1993)

\section{9 .4 \\ Méthode}

Le dispositif mis en place par ITGN (réseau de microprismes réflecteurs) a été complété à partir de juin 1993 
avec 13 repères identifiables sur des clichés aériens. Ces repères ont été disposés dans la zone médiane et leur position déterminée par GPS. Cette extension du réseau avait un objectif multiple: élargir le dispositif de suivi, servir de base à une stéréopréparation pour l'exploitation photogrammétrique de vues aériennes, et étalonner les déformations que des traitements numériques de ces clichés allaient mettre en évidence.

\section{8,2 \\ Résultats}

Les mesures GPS réalisées par la socièté Sintégra montrent:

- un net ralentissement du glissement entre juin et novembre 1993:

\section{$2,2 \leq \mathrm{v} \leq 3,3 \mathrm{~mm} / \mathrm{i}$, soit 0,8 à $1,6 \mathrm{~m} / \mathrm{an}$;}

- une accélération de novembre 1993 à mars 1994:

$$
6,4 \leq \mathrm{v} \leq \text { à } 12,2 \mathrm{~mm} / \mathrm{j} \text {, ou } 2,3 \text { à } 4,5 \mathrm{~m} / \mathrm{an} \text {; }
$$

- un ralentissement entre mars et avril 1994, avec des valeurs comparables à celles mesurées entre juin et novembre (1993) :

\section{$2,8 \leq v \leq 4,1 \mathrm{~mm} / \mathrm{j}$, soit de 1 à $1,5 \mathrm{~m} / \mathrm{an}$.}

Le mouvement général s'effectue vers le SW (direction N 200 à N 225). Les variations des vitesses de déplacement correspondent à celles mesurées par visées optiques.

Les mesures GPS, comme l'auscultation topométrique, révèle une grande variabilité de la cinématique du glissement, certainement en partie liée aux conditions pluviométriques. Deux importantes crises (maiaoût 1992 et novembre 1993-mars 1994) caractérisées par des vitesses très élevées, sont mises en évidence.

Il est probable que le glissement du panneau calcaire soit un mouvement continu: les mesures obtenues entre janvier 1992 et avril 1994 suggèrent qu'un mouvement de fond s'est produit à une vitesse comprise entre 1 et $1,5 \mathrm{~m} / \mathrm{an}$.

A cette époque, une extension des désordres au sud de la croupe de Mondorès a été observée.

\section{3}

\section{Exploitation des MNT et des ortho-images}

\subsection{4}

\section{Principe}

La méthode est basée sur la comparaison de MNT et d'images ortho-rectifiées, numériques, issus d'une analyse photogrammétrique des clichés aériens de 1972 (échelle 1/30000), 1991 et 1993 (échelle 1/8000). Les ortho-images ont une résolution planimétrique de $0,50 \mathrm{~m} \times 0,50 \mathrm{~m}$, les MNT de $1 \mathrm{~m} \times 1 \mathrm{~m}$. L'incertitude sur le calage altimétrique de points de référence au sol est de $3 \mathrm{~m}$. Les différences observées entre les MNT révèlent la composante verticale du champ des déformations superficielles du versant. La comparaison de couples (MNT, ortho-image) permet de déterminer la position de points remarquables, identifiés sur les clichés aux différentes dates; les vecteurs de déplacement qui peuvent alors être définis montrent l'amplitude et la direction des mouvements de surface.

\section{$233: 9$}

\section{Résultats}

\section{- Évolution de la zone médiane}

Les différences entre les MNT (Fig. 2 et 3 ) révèlent: - un net creusement de la zone médiane avec un volume déficitaire d'environ $300000 \mathrm{~m}^{3}$ entre 1972 et 1991, et de près de $45000 \mathrm{~m}^{3}$ entre 1991 et 1993 ;

- un soulèvement important (de plusieurs mètres) de la croupe de Mondorès. Cette croupe présente, sur le terrain, de nombreuses crevasses, des arrachements et des affaissements qui témoignent d'une désorganisation avancée;

- l'existence d'une zone de compression, orientée sensiblement N-S, parallèle au fossé de Terre-Rouge Mondorès :

- des mouvements ascendants à l'extrémité sud de cette zone, contre la croupe de Mondorès, confirmés par ailleurs par GPS.

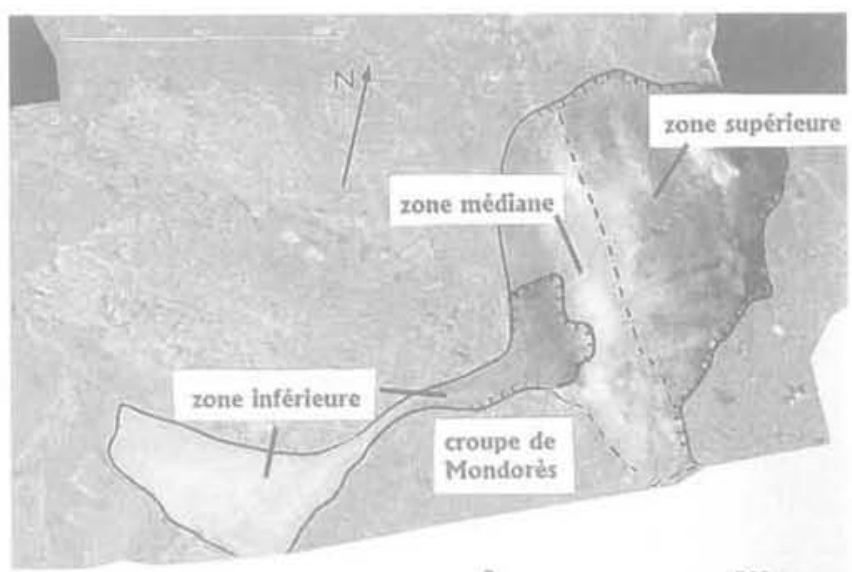

0 $500 \mathrm{~m}$

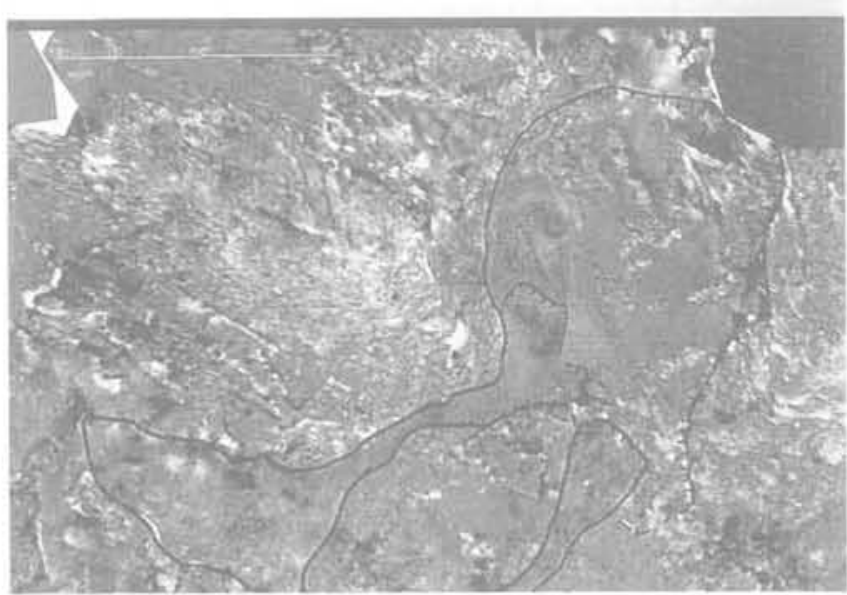

FG. 2 Image des variations altimétriques entre les MNT de 1972 et 1993 (haut) et de 1991 et 1993 (bas); expression graphique: gris clair : élévation, gris foncé: abaissement de la surface topographique.

Substraction of DEM images [1972 and 1993 (top): 1991 and 1993 (bottom)] graphic expression: light grey: uplift, dark grey: subsidence. 

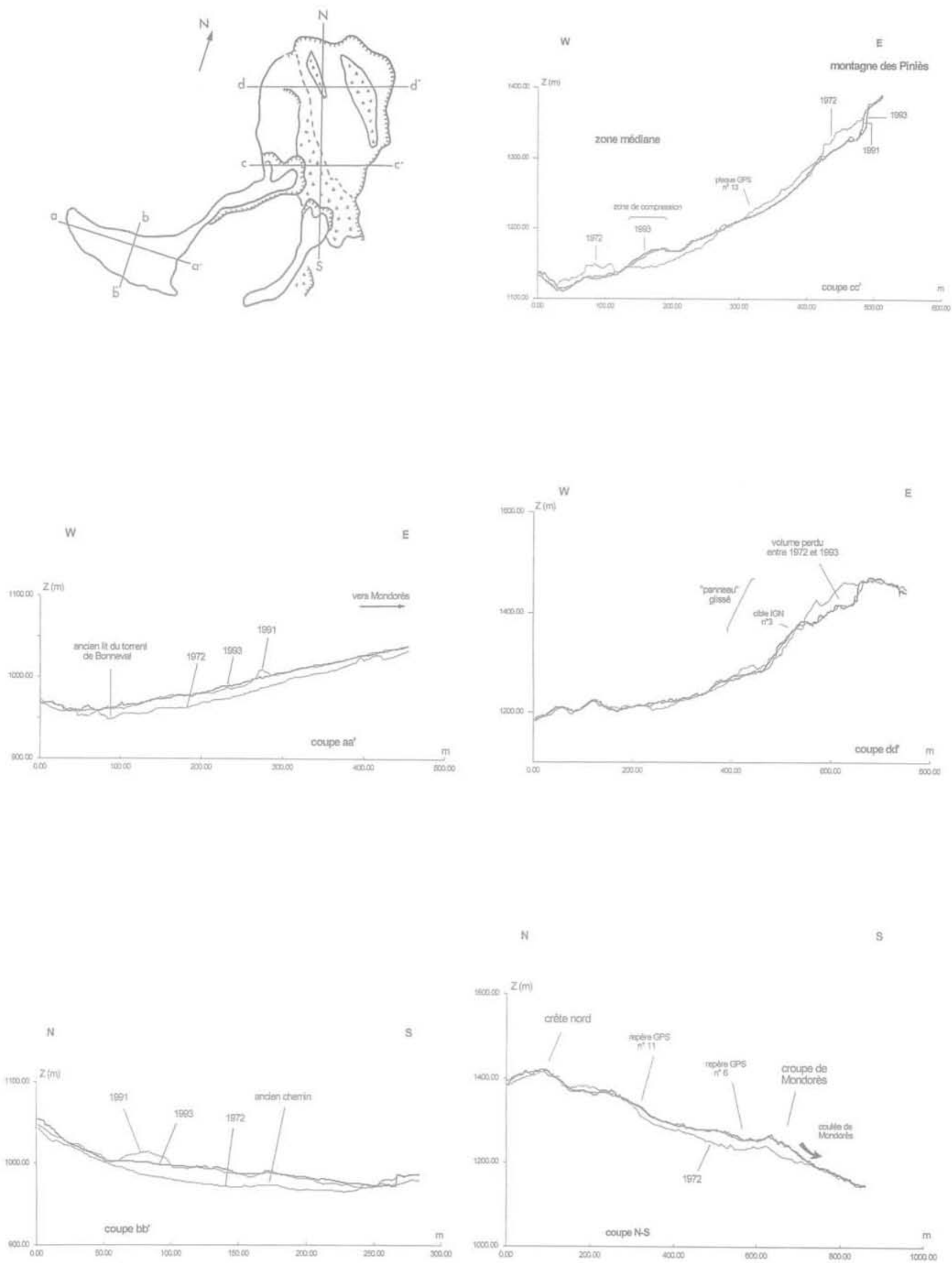

FG, 3 Profils comparatifs réalisés à partir des MNT et schéma de localisation. Comparative cross sections, extracted from the DEMs. 


\section{- Évolution de la zone inférieure}

Des profils topographiques comparatifs réalisés à partir des MNT (Fig. 3) représentent l'évolution du cône d'accumulation des coulées de Taravel et celle des zones médiane et supérieure. Une évaluation quantitative du volume de ce cône a été effectuée. Elle révèle un engraissement d'environ $815000 \mathrm{~m}^{3}$, un volume calculé proche de celui, déficitaire, du fossé qui sépare la montagne des Piniès du compartiment qui s'en détache $\left(829000 \mathrm{~m}^{3}\right.$ environ). L'épaisseur moyenne du cône est donc de $11 \mathrm{~m}$; elle peut atteindre, localement, $20 \mathrm{~m}$.

- Le mouvement de points singuliers identifiés sur les clichés

Il s'agit de points facilement repérables (pointements rocheux, blocs, ressauts, etc.) identifiés à la fois sur les clichés de 1991 et de 1993. Les bouleversements antérieurs n'ont pas permis de reconnaître formellement de tels points entre 1972 et 1991.

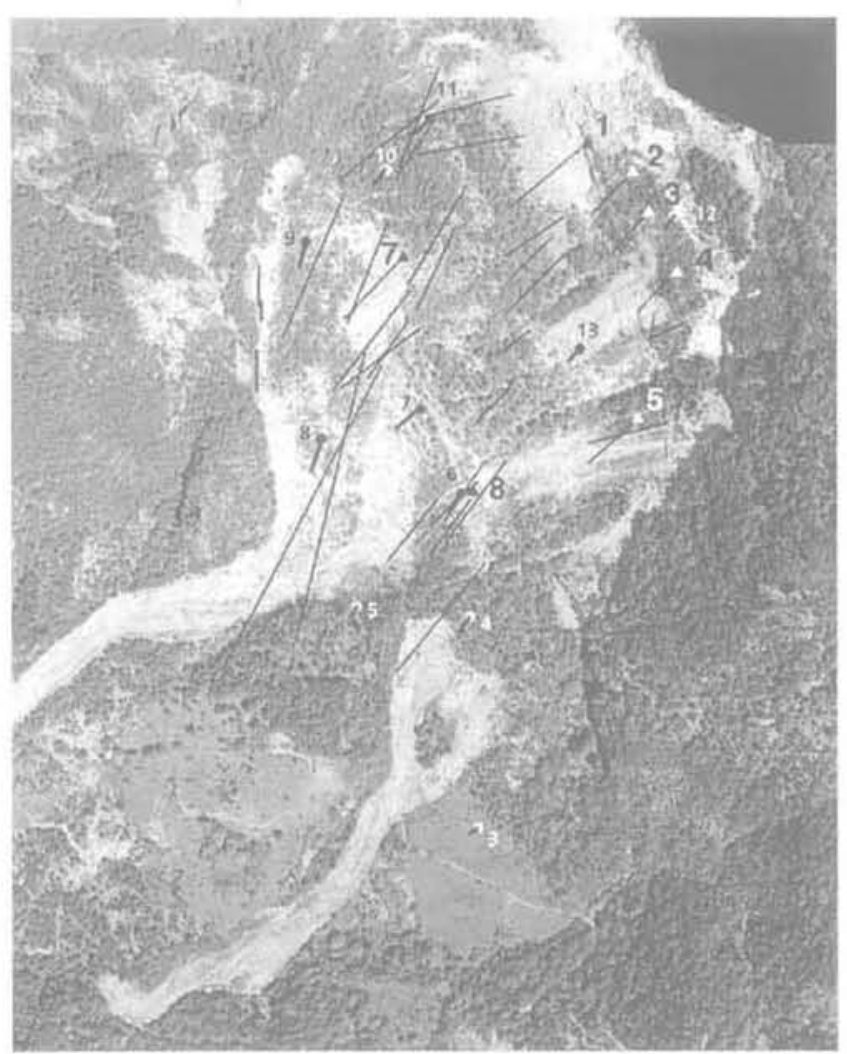

$F \mid$ I 4 Image des mouvements plans $(x 20)$ dans les zones médiane et supérieure (en direction du SW, ou du sud) déterminés par les différentes méthodes -micro-réflecteurs IGN (auscultation topométrique, 27/01/92 à 28/06/93)

- plaques GPS (11/06/93 à 15/11/93) - points naturels remarquables identifiés sur les ortho-images du 14/12/1991 et du 15/07/1993.

Horizontal component of movements $(\times 20)$ toward the SW and S, in intermediate and upper zones, according to the different methods

-microreflectors of IGN (topometric

auscultation)

- GPS targets

- natural, remarquables spots of 1991 and 1993 ortho-images.
Sur la figure 4 ont été reportés, outre les déplacements des réflecteurs IGN et des repères GPS, ceux de 28 points singuliers. Le déplacement vers le SW des masses supérieures affaissées et des matériaux de surface de la zone médiane est clairement représenté. Ceux-ci sont déviés à l'aval par l'éperon 1180. Il apparait nettement que le mouvement, qui affecte des terrains mal connus, est complètement généralisé.

\section{4}

\section{Expérimentation DORIS}

Principe

Le BRGM et la société CLS (Collecte Localisation Satellite) ont testé le système de localisation par satellite DORIS (Doppler Orbitography and Radiopositioning Integrated by Satellite). Ce système permet de connaître avec une grande précision les paramètres orbitaux des satellites à bord desquels il est embarqué (Spot, TopeixPoseidon). En retour, les coordonnées de stations radio émettrices sont calculées avec la même précision $(10 \mathrm{~cm}$ en coordonnées absolues, $3 \mathrm{~cm}$ en coordonnées relatives). L'intérêt de la méthode, outre sa précision, réside dans la fréquence des mesures de positionnement: les balises sont ( interrogées») chaque fois que le satellite est en vue du site: en moyenne 3 fois par jour à la latitude de Boulc. Le calcul des coordonnées est effectué et affiné au cours du temps par une moyenne glissante sur des périodes de mesures de plusieurs jours (2 à 5 dans le cas de cette expérimentation, 10 à 15, voire plus, si la précision souhaitée est plus grande).

Afin de tester ce système, deux prototypes de balises de radio-positionnement ont été installés dans la zone médiane (balise A) et dans la zone inférieure (balise B, située dans la partie supérieure du cône d'accumulation des coulées). Pour éviter leur éventuel enfoncement dans les terrains meubles, les deux prototypes reposaient sur une structure flottante (socle) en contre-plaqué. Afin de valider la méthode et la fiabilité des données, 1TGN avait intégré ces balises dans son réseau de surveillance topométrique. Les mesures ont été réalisées entre juillet et août 1992.

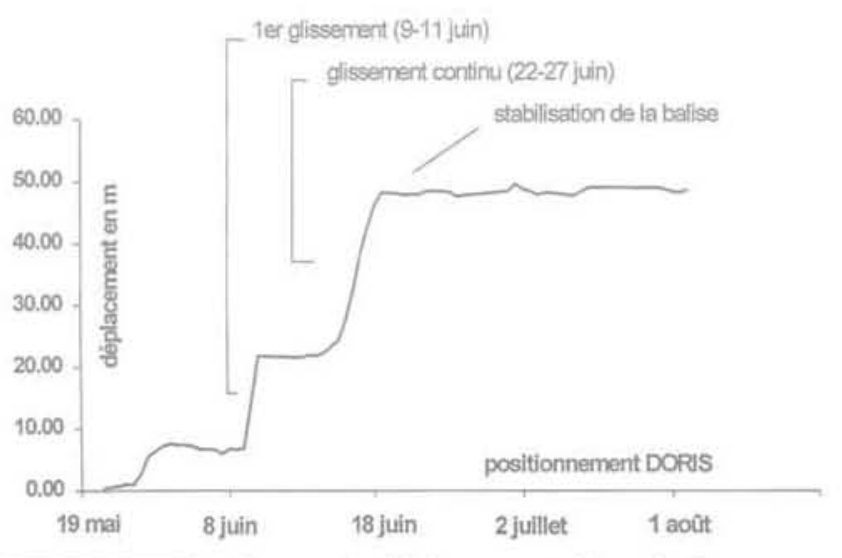

FiG,5 Mesure du déplacement d'une balise radio-émettrice par le système DORIS. Measurements of a beacon's motion using the DORIS system. 


\section{Résultats}

Au cours de l'expérimentation, la balise A, implantée au-dessus de la zone d'alimentation des coulées, a présenté d'importantes oscillations, sans déplacement, qui témoignent de l'activité intense de cette zone. Pour mieux comprendre la dynamique de cette activité et la corréler avec l'émission possible de coulées boueuses, il aurait été intéressant de disposer de données météorologiques et d'observations plus fréquentes du terrain. Il n'a pas été possible de les réaliser à cette époque. Les balises actuelles, miniaturisées, plus perfectionnées, sont capables de transmettre des données de pluviométrie.

La balise B, emportée par une coulée de boue, a été déplacée sur plusieurs dizaines de mètres. Ses déplacements successifs ont été mesurés (Fig. 5) et son mouvement cartographié.

A l'issue de l'expérimentation, les deux stations ont été récupérées en bon état. L'incertitude sur les mesures, de l'ordre de la dizaine de centimètres en coordonnées absolues, montre que le système DORIS est adapté à l'analyse et au suivi tridimensionnel de déformations rapides et importantes au sein de versants instables.

\section{3}

\section{Conclusions et perspectives}

Les auscultations réalisées entre 1992 et 1993 sur ce glissement de grande ampleur, au moyen de techniques traditionnelles (topométrie par visées optiques) ou récentes (positionnement GPS, localisation DORIS) permettent de mieux connaître son activité et son évolution. Les diverses données mettent en évidence l'évolution de la zone médiane et l'affaissement du panneau détaché de la montagne des Piniès.

Les suivis topométriques et GPS montrent la variabilité, dans le temps, des vitesses de déplacement.
La comparaison des images numériques de 1991 et 1993 fournit des informations qualitatives et quantitatives sur l'ensemble du glissement, et permet, avec une résolution de $1 \mathrm{~m}^{2}$ :

- de cartographier dans sa continuité spatiale le champ des déformations superficielles;

- d'extraire et de comparer de nombreux profils topographiques:

- d'identifier en tout point des vecteurs de déplacement.

Il n'a toutefois pas été possible d'étalonner les déformations mises en évidences par la comparaison de MNT. Les plus petites d'entre elles paraissent être de l'ordre de la dizaine ou de quelques dizaines de centimètres. Les mesures GPS, complétant les observations de terrain, confirment le soulèvement, détecté sur les images numériques, de la zone comprimée près de la croupe de Mondorès. Les vecteurs de déplacement prouvent enfin que le glissement est généralisé et qu'il se produit vers le SW.

Les balises DORIS ont révélé une activité intense de la zone médiane, difficile à mesurer par ailleurs. Cette expérimentation laisse entrevoir de nouvelles possibilités pour assurer le suivi de l'évolution de versants instables ou pour mesurer pratiquement en continu des déformations importantes et rapides. Des positionnements plus précis, de l'ordre de quelques centimètres, sont obtenus en coordonnées relatives (par rapport à une balise fixe située à proximité du site). La continuité des mesures permet de rechercher d'éventuels liens entre l'activité d'un glissement et les conditions pluviométriques.

\section{Remerciements}

Cet article est la publication scientifique n $96001 \mathrm{du}$ BRGM; il a été réalisé dans le cadre d'un projet financé sur crédits de recherches du BRGM auquel a participé, dans le cadre d'un projet de Recherche en Partenariat Industriel, la Société CIS.

\section{Bibliographie}

Girault F., Goguel B., Asté J.-P. - Le glissement du Friolin, en Savoie : mesures par imagerie numérique, Revue Francaise de Géotechnique, n 73, p. 73-79, 1995

Girault F. Fleury B. - Photogrammetry and digital imagery : application to measuring and defining deformations in unstable slopes, Proceedings of the 1st International Airborne Remote Sensing
Conference and Exhibition (Strasbourg. France, vol. III, p. 198-204. 1994.

Girault F. - Auscultation de versants instables par imagerie numérique; thèse Ing. CNAM, Paris, 193 p, 1992

Malatrait A.-M., Sabatier F. - Le glissement de la montagne des Piniès à l'origine des coulées de Boulc-en-Diiois (Drôme). Évolution et mécanismes. Revue Fran- çaise de Gẻotechnique n*75, p, 45-53, 1996.

Malatrait A.-M - Le glissement de la montagne des Piniès à l'origine des coulées de Boulc-en-Diois (Dróme), journées thématiques du 22/03/95. Glissements de terrain et grands rem. blais, INSA de Toulouse, 6 p. 1995. 